\title{
A Tribute to Max von Laue
}

Henk Kubbinga,

University of Groningen (The Netherlands)

DOI: $10.1051 /$ epn/2012602

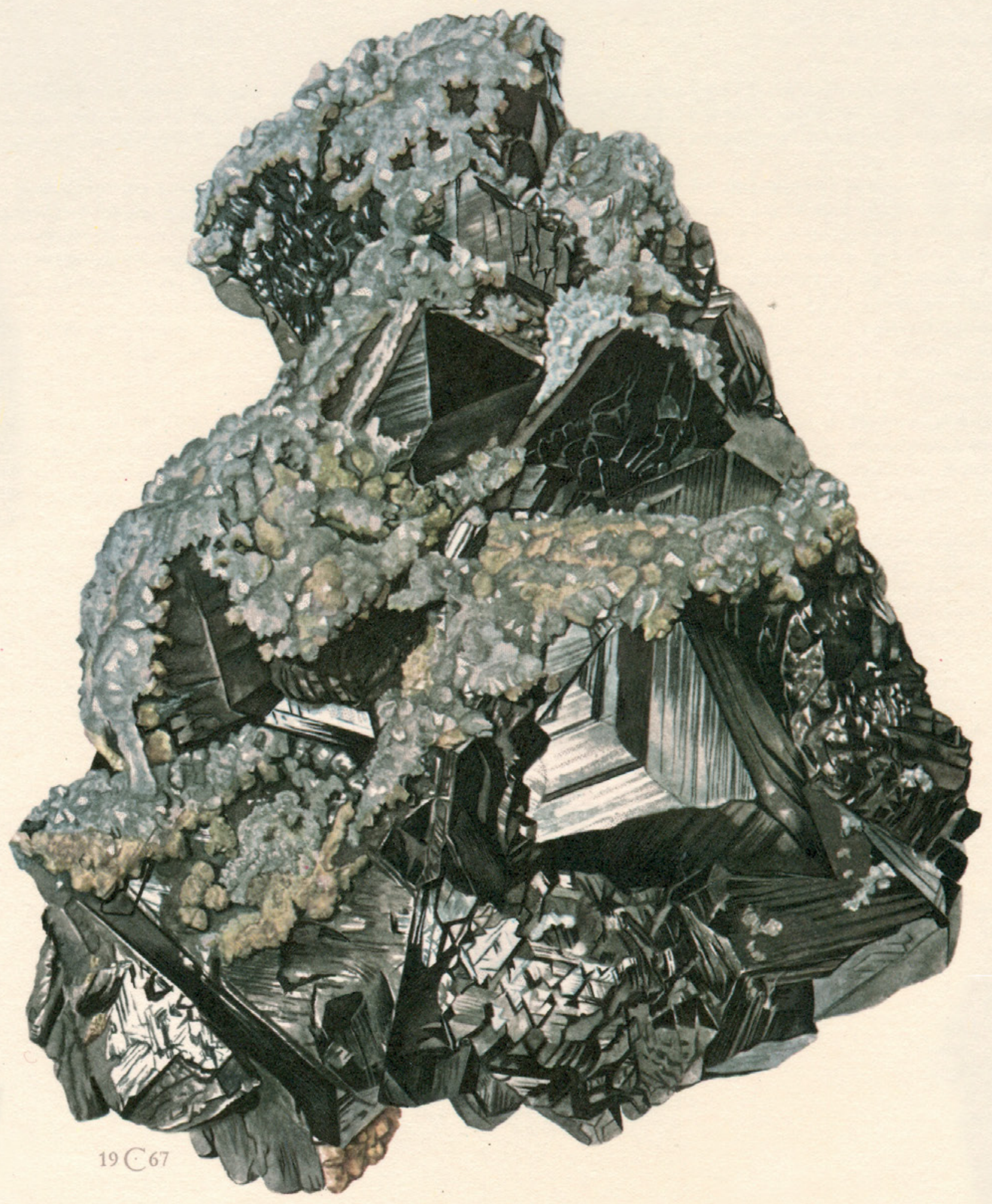


Just an idea: take a crystal, surround it with photographic plates, and direct a pencil of X-rays at it, perpendicularly. It was one of those thrilling ideas that made new science. Max Laue was the proponent, in April 1912. The experiment as such had been tried out before by Röntgen himself, but without avail. Friedrich and Knipping, however, took their time and waited long enough. Overnight, the atomic variant of lattice theory-which had worked its way at the outskirts of crystallography_became fashionable. And, not to forget, $X$-rays proved to be wave-like indeed.

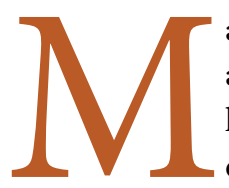

ax Laue originated from Pfaffendorf, a village now part of Koblenz. After his Abitur he enrolled at the university of Strasbourg, moving on, in 1899, to Göttingen, the place to be for theoreticians. Optics became his favourite subject. Waldemar Voigt and Otto Lummer showed the way to go. His PhD subject brought him to Berlin to study with Max Planck. In 1903 Laue defended a thesis about the diffraction which occurs during interference caused by plane parallel plates. It was Planck who asked him, in 1906, to become his assistant. Laue's Habilitation assessed the entropy of interfering pencils of rays. It was the time that one Albert Einstein's freshly appeared paper 'Zur Elektrodynamik bewegter Körper' was favorably discussed by Planck in a lecture at the Colloquium of Berlin's Physical Society.

\section{Relativity: theory and experiment}

Laue was in the audience. It took some time, but his conversion was unconditional. He went to see Einstein in Switzerland, soon after his Habilitation, to discuss details. His was one of the first experimental arguments if not proofs (1907), in the sense that he identified Einstein's 'addition theorem' with a formula derived by Fizeau (1851) for the velocity of light in flowing water. Fizeau, it is recalled, first established (together with Foucault) that in denser media light is slowed down-in conformity with the wave-theory-while the motion of some such medium may be used to move an interference pattern: the fringes produced by coherent light led through the two arms of a water circuitry slightly shifted when the flow direction was changed. Fizeau did not insist and left others the task to improve his method. It was Michelson and Morley who did so in 1887. There were some problems with the turbulence of the flowing water and Pieter Zeeman, therefore, used a movable glass stick instead. Laue produced the first general account of the recent developments in Das Relativitätsprinzip (1911) and succeeded in familiarizing many an estranged physicist with the broad context-and the paradoxes-of what came to be known as special relativity. In 1920 he would publish, as a supplement, a similar account of Einstein's latest breakthrough, general relativity.

\section{Rays and rays and... X-rays}

At the turn of the XIX ${ }^{\text {th }}$ and $\mathrm{XX}^{\text {th }}$ century a whole series of seemingly distinct, though more or less related radiation phenomena disputed each other's priority in the physicists' attention. One of the most enigmatic was, without doubt, the one revealed by Wilhelm Röntgen in the fall of 1896. For the general public, however, it had been overshadowed, by some natural rays, spontaneously emitted by materials that could be concentrated by chemical means. Laue got involved in 1909 when he entered, as a Privatdozent, in the service of Arnold Sommerfeld, at the University of Munich. Sommerfeld himself had been nominated a few years before to provide the theoretical background to Röntgen's rays. Röntgen was still around, but left Sommerfeld and the latter's students free to use his instruments, among which a $50 \mathrm{~cm}$ Rühmkorff-inductor and a whole collection of bulbs.

\section{Lattice theory in crystallography}

The $19^{\text {th }}$ century had lived with the rise of lattice theory. Crystals had been conceived of, by René-Juste Haüy (1743-1822), as well-ordered 'assemblages' of polyhedral

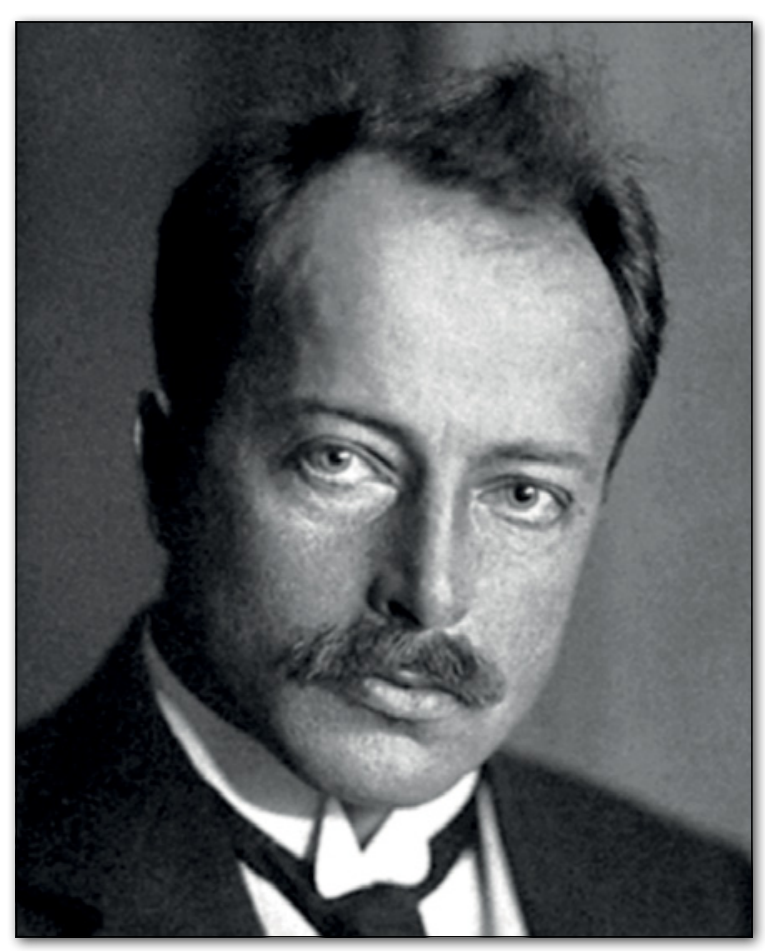

P.18: Blende (ZnS; covered with calcite) from Trepca (Serbia). Aquarelle by Claus Caspari (1911-1980). Reproduced from H. Schröcke and K.I. Weiner, Mineralien (Hamburg: E. (ramer, 1967).
4 FIG. 1: Max von Laue (1920; courtesy Nobel Foundation). 
molecules composed of the atoms of the elements. Those of pyrite, for instance, were presented as 'assemblages' of cubic $\mathrm{FeS}_{2}$-molecules. Such 'assemblages' could take the form of a cube or orthogonal parallellepiped, naturally, but also that of a dodecahedron or octahedron, depending on the regularities during the growth. It was a personal triumph for Haüy not only that the interfacial angles could be calculated in advance, but also that they agreed closely with those measured on natural crystals, for instance, in the case of dodecahedral pyrite. His successors gradually replaced the molecules by their centres of gravity. In this way the crystal became an abstract point lattice whose symmetry properties defied the imagination of men like Delafosse, Bravais and Jordan. Particular compounds, like the alums, though, suggested the existence of superimposed molecular lattices: after all, the crystal water of these so-called hydrates could be easily separated from the rest, while a crystal of the original form emerged on evaporating a solution. It was Leonhard Sohncke (1842-1897) who concluded, in 1888 , that any crystal could be considered as an array of as many lattices as there are atomic species in it. In the 1890's

v FIG. 2: The original set-up as used by Friedrich and Knipping to see the effect of X-rays on a copper sulfate crystal. From left to right on the table: Röntgen bulb (too low here), first diaphragm (lead foil), collimator, round turntable with the crystal at its centre and an enveloped photographic plate. A huge wooden tripod is used to rise and reset a shielding cover of lead foil. Since 1921 this set-up is part of the collection of the Deutsches Museum, München (courtesy: Deutsches Museum).
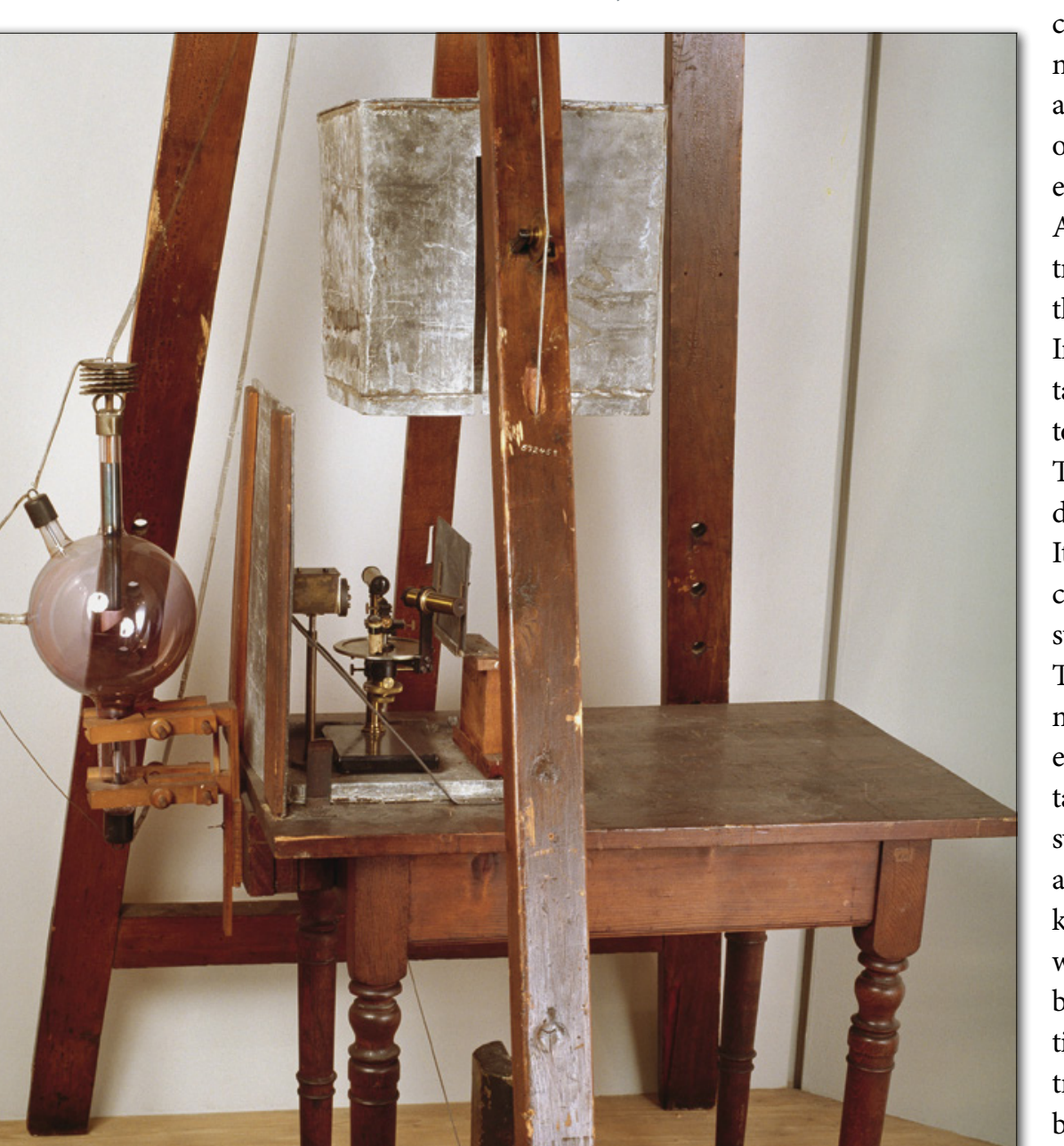
$\left[97.4 /\left\{4.06 \times\left(6.17 \times 10^{23}\right)\right\}\right]^{1 / 3}=3.38 \times 10^{-8} \mathrm{~cm}$. This would be the lattice constant. It brought Laue, Friedrich and Knipping instantaneous fame. In 1913 and, again, in 1914, Laue was nominated for the Nobel Prize for Physics. There were but few nominations, it is true, but these were enough for the Selection Committee to decide in 1914 in favor of Laue. In the 1930's Laue, in his position of chairman of the German Physical Society and deputy director of the KaiserWilhelm Institute for Physics, got involved in politics, like any other German. His fierce struggle against the nomination of Johannes Stark, a fervent Nazi, at various powerful positions became emblematic for civil courage. These were also the days that he favoured the development of a new, extremely promising visualisation technique for submicron entities: the electron microscope. After the war this technique would feature among the first subjects to be launched in the new German physics.

\section{Acknowledgment}

It is a pleasure to thank Wolfgang Schmahl (Director of the Crystallography Department of Ludwig-MaximiliansUniversität, Munich) and Wolfgang Heckl (Director of the Deutsches Museum) for their hospitality and technical support.I am obliged to Karl Grandin (Director of the Archives of the Royal Swedish Academy of Sciences) for kindly allowing me to consult the nomination letters for Laue.

\section{About the author}

Henk Kubbinga is a historian of science at the University of Groningen and member of the EPS History of Physics Group. He is currently editing The Collected Papers of Frits Zernike (1888-1966), the first two volumes of which were recently published by Gröningen University Press (ISBN 978-90-814428-3-1).

\section{Notes and References}

For the broad context see Acta Crystallographica A 68 (1) (2012) and Zeitschrift für Kristallographie 227, 1 (2012), a joint issue edited by Wolfgang Schmahl and Walter Steurer.

[1] M. Laue, 'Wellenoptik', in: F. Klein and Sommerfeld (eds.) Encyklopaedie der mathematischen Wissenschaften, mit Einschluss ihrer Anwendungen, Leipzig: Teubner, 1898-1926, volume V.3, 362 (finished by July 1915).

[2] W. Friedrich, P. Knipping and M. Laue, Sitzungsberichte (München) 1912, 303.

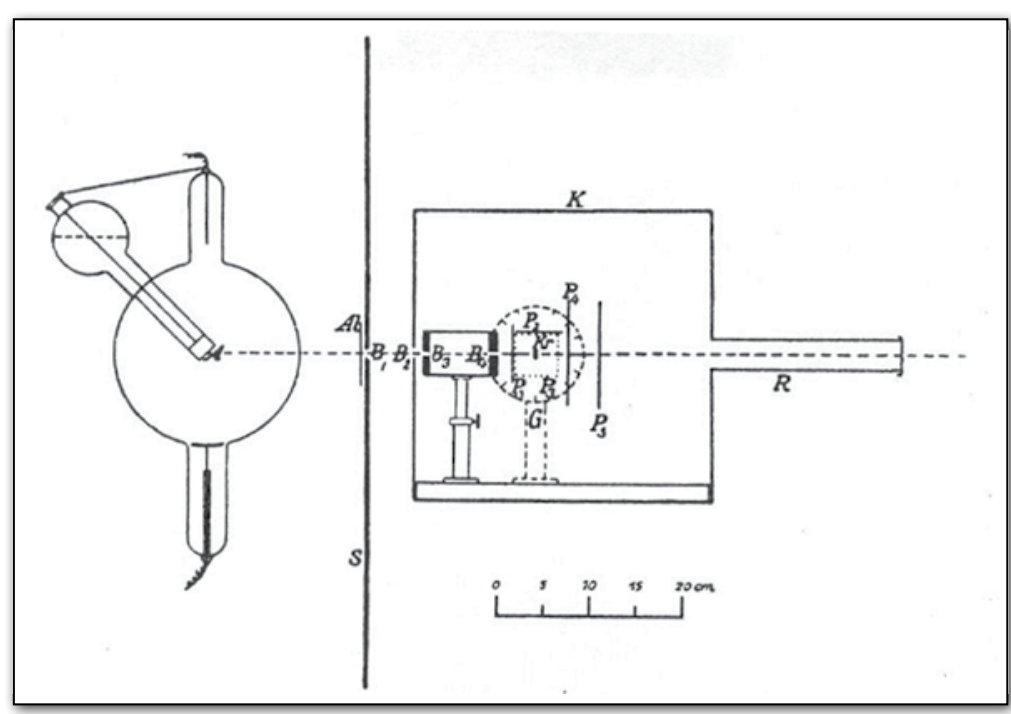

$\triangle$ FIG. 3: Final set-up of Friedrich, Knipping and Laue as used for the 001 platelet of ZnS. The Röntgen bulb is now watercooled. The platelet is glued at $\mathrm{Kr}$ on a finely tunable round turntable $\mathrm{G}$ (seen from above; interrupted line), with photographic plates at P1-5. Successive diaphragms, B1-4, narrow down the beam coming from anti-cathode $A ; R$ is a tube-of-no-return (from ref.[2]).

V FIG. 4: The first photogram of an 001 platelet of ZnS as made by Friedrich, Knipping and Laue (from ref. [2]).

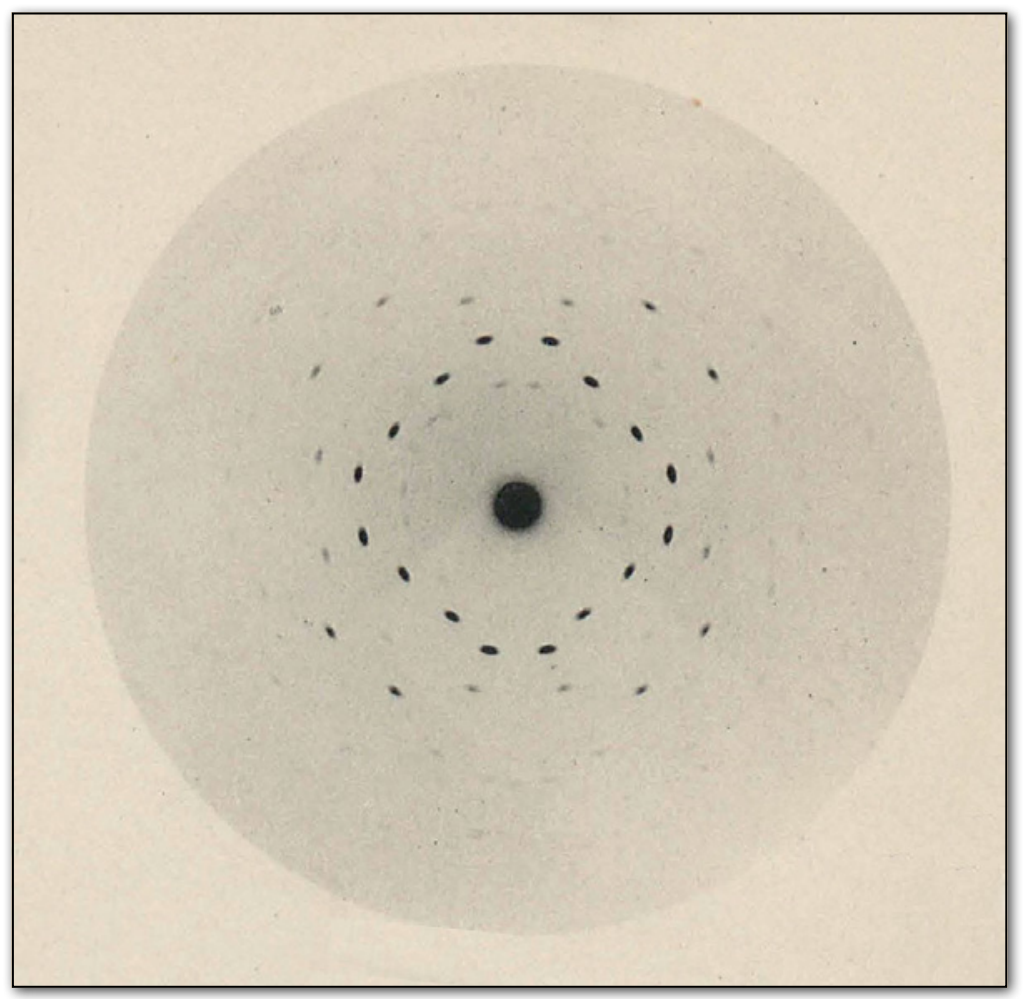

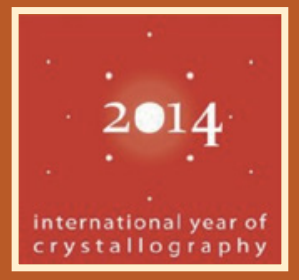

\section{4: INTERNATIONAL YEAR OF CRYSTALLOGRAPHY}

The UN decision was announced on 4 July 2012 by the International Union of Crystallography (IUCr), which since several years has been proposing to mark with an International Year the centenaries of www.iucr.org n the discovery of X-ray diffraction by Max von Laue in 1912 and of the formulation of Bragg's Law in 1913. For more information, see the Website of the IUCR: 